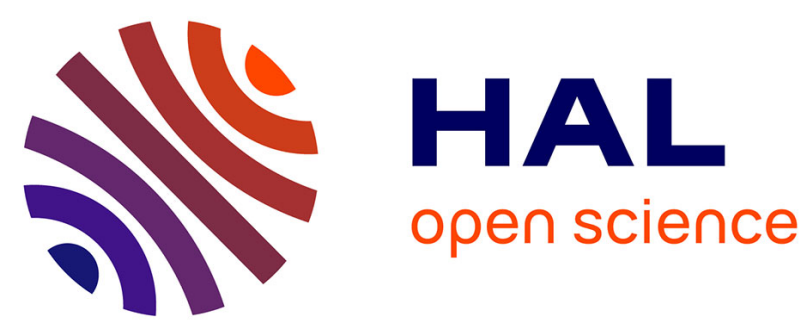

\title{
Application of the PGD and DEIM to solve a 3D Non-Linear Magnetostatic Problem coupled with the Circuit Equations
}

\author{
Thomas Henneron, Stéphane Clenet
}

\section{- To cite this version:}

Thomas Henneron, Stéphane Clenet. Application of the PGD and DEIM to solve a 3D Non-Linear Magnetostatic Problem coupled with the Circuit Equations. IEEE Transactions on Magnetics, 2015, 52 (3), pp.1-4. 10.1109/TMAG.2015.2487539 . hal-01273222

\section{HAL Id: hal-01273222 \\ https://hal.science/hal-01273222}

Submitted on 12 Feb 2016

HAL is a multi-disciplinary open access archive for the deposit and dissemination of scientific research documents, whether they are published or not. The documents may come from teaching and research institutions in France or abroad, or from public or private research centers.
L'archive ouverte pluridisciplinaire HAL, est destinée au dépôt et à la diffusion de documents scientifiques de niveau recherche, publiés ou non, émanant des établissements d'enseignement et de recherche français ou étrangers, des laboratoires publics ou privés. 


\title{
Application of the PGD and DEIM to solve a 3D Non-Linear Magnetostatic Problem coupled with the Circuit Equations
}

\author{
T. Henneron ${ }^{1}$, S. Clénet ${ }^{2}$ \\ ${ }^{1}$ L2EP, Université Lille 1, 59655 Villeneuve d'Ascq, France \\ ${ }^{2}$ L2EP, Arts et Métiers ParisTech, 59046 Lille, France
}

\begin{abstract}
Among the model order reduction techniques, the Proper Generalized Decomposition (PGD) has shown its efficiency to solve static and quasistatic problems in the time domain. However, the introduction of nonlinearity due to ferromagnetic materials for example has never been addressed. In this paper, the PGD technique combined with the Discrete Empirical Interpolation Method (DEIM) is applied to solve a non-linear problem in magnetostatic coupled with the circuit equations. To evaluate the reduction technique, the transient state of a three phase transformer at no load is studied using the full Finite Element model and the PGD_DEIM model.
\end{abstract}

Index Terms-Proper Generalized Decomposition, Empirical Interpolation Method, Non-Linear magnetostatic problem

\section{INTRODUCTION}

$\mathrm{T}_{\mathrm{n}}$ O reduce the computation time of time-dependent numerical models, Model Order Reduction (MOR) methods have been developed and presented in the literature. These methods consist in searching a solution in a subspace of the approximation space of the full numerical model [1][2]. They have been mainly used to solve problems in mechanics. In this field, the Proper Generalized Decomposition (PGD) method has been developed since the early 2000's and knows an increasing interest in the scientific community [3][4]. For problems in the time domain, the PGD method consists in approximating the solution by a sum of separable functions in time and space, so-called modes. Each mode is determined by an iterative procedure and depends on the previous modes. In the case of non-linear problems, the MOR methods are not so efficient than in the linear case, due to the computation cost of the non-linear terms. In fact, the calculation of the non-linear terms of the reduced model requires the calculation of the nonlinear vectors or/and matrices of the full model. To circumvent this issue, the Discrete Empirical Interpolation Method (DEIM) method can be used [5][6]. This method consists in interpolating the non-linear terms of the full model by calculating only some of their entries. In the literature, the PGD approach has been combined with the DEIM in order to solve a thermal problem with a quadratic nonlinearity [7]. In computational electromagnetics, the PGD approach has been developed to study a fuel cell polymeric membrane model [8].

In static electromagnetism, the behavior of a Soft Magnetic Composite Material has been modeled [9]. In the case of magneto-quasistatics, the skin effect in a rectangular slot or in a conducting plate has been addressed [10][11]. However, any non-linear problem has not been solved using the PGD combined with the DEIM in computational electromagnetics.

In this paper, we propose to apply the PGD_DEIM approach to solve a 3D non-linear magnetostatic problem coupled with multiple external electric circuits using the vector potential formulation. First, the non-linear magnetostatic problem coupled with electric circuits is presented. Secondly, the PGD_DEIM approach is developed.
Finally, a three phase transformer at no load is studied in the case of a sinusoidal supply and also with a PWM supply. The results obtained with the PGD_DEIM model are compared in terms of accuracy and computation time with the full model.

\section{NON-LINEAR MAGNETOSTATIC PROBLEM COUPLED WITH ELECTRIC CIRCUIT EQUATIONS}

Let us consider a domain D of boundary $\Gamma\left(\Gamma=\Gamma_{\mathrm{B}} \cup \Gamma_{\mathrm{H}}\right.$ and $\Gamma_{\mathrm{B}} \cap \Gamma_{\mathrm{H}}=0$ ) (Fig. 1). The problem is solved on $\mathrm{D} \times[0, T]$ with $T$ the width of the time interval. The eddy current effect is neglected however several stranded inductors are considered.

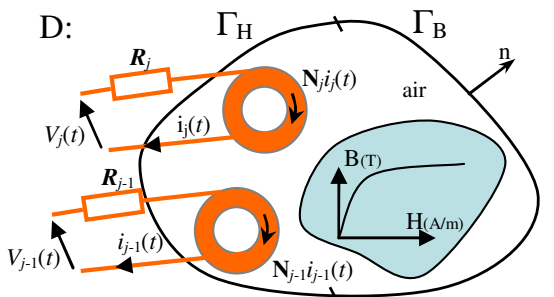

Fig. 1. Non-linear magnetostatic problem coupled with electric circuits

In the case of magnetostatics, the problem can be described by the following equations:

$$
\begin{aligned}
& \operatorname{curl} \mathbf{H}(\mathbf{x}, t)=\sum_{j=1}^{N_{s t}} \mathbf{N}_{j}(\mathbf{x}) i_{j}(t) \\
& \operatorname{div} \mathbf{B}(\mathbf{x}, t)=0 \\
& \mathbf{H}(\mathbf{x}, t)=\mathrm{v}_{(\mathbf{B})}(\mathbf{x}) \mathbf{B}(\mathbf{x}, t)
\end{aligned}
$$

where $\mathbf{B}$ is the magnetic flux density, $\mathbf{H}$ the magnetic field, $\mathbf{N}_{\mathbf{j}}$ the unit current density vector and $i_{j}$ the current flowing through the $j^{\text {th }}$ stranded inductor, $N_{s t}$ is the number of inductors and $v_{(\mathbf{B})}(\mathbf{x})$ is the reluctivity which depends on $\mathbf{B}$ in the ferromagnetic part. To impose the uniqueness of the solution, boundary conditions are introduced such that:

$$
\mathbf{B}(\mathbf{x}, t) \cdot \mathbf{n}=0 \text { on } \Gamma_{\mathrm{B}} \text { and } \mathbf{H}(\mathbf{x}, t) \times \mathbf{n}=0 \text { on } \Gamma_{\mathrm{H}}
$$

with $\mathbf{n}$ the outward unit normal vector. In order to impose the voltage at the terminals of the stranded inductors, the following relations are added: 


$$
\frac{\mathrm{d} \Phi_{j}(t)}{\mathrm{dt}}+R_{j} i_{j}(t)=v_{j}(t) \text { with } j=1, . ., N_{s t}
$$

where $R_{j}$ is the resistance, $\Phi_{j}$ the flux linkage and $v_{j}$ the voltage of the $j^{\text {th }}$ inductor. To solve the problem, the vector potential formulation is introduced. The vector potential $\mathbf{A}$ is defined such that $\mathbf{B}(\mathbf{x}, t)=\mathbf{c u r l} \mathbf{A}(\mathbf{x}, t)$ with $\mathbf{A}(\mathbf{x}, t) \times \mathbf{n}=0$ on $\Gamma_{\mathrm{B}}$. To take into account the non-linear behavior of the ferromagnetic materials, the magnetic field $\mathbf{H}(\mathbf{x}, t)$ is defined by $\mathbf{H}(\mathbf{x}, t)=v_{\mathrm{fp}} \mathbf{B}(\mathbf{x}, t)+\mathbf{H}_{\mathrm{fp}}(\mathbf{B}(\mathbf{x}, t))$ with $v_{\mathrm{fp}}$ a constant and $\mathbf{H}_{\mathrm{fp}}(\mathbf{B}(\mathbf{x}, t))=\left(v_{(\mathbf{B})}(\mathbf{x})-v_{\mathrm{fp}}\right) \mathbf{B}(\mathbf{x}, t)$ a virtual magnetization vector. In the materials with a constant reluctivity $v$, the same expression can be used with $v_{\mathrm{fp}}=v$ and $\mathbf{H}_{\mathrm{fp}}(\mathbf{B}(\mathbf{x}, t))=0$. To determine a solution to the problem on $\mathrm{D} \times[0, T]$, weak forms of (1) and (5) can be written such that:

$$
\begin{gathered}
\int_{0}^{T} \int_{\mathrm{D}} v_{\mathrm{fp}} \operatorname{curl} \mathbf{A}(\mathbf{x}, t) \cdot \operatorname{curl} \mathbf{A}^{\prime}(\mathbf{x}, t) \mathrm{dDd} t \\
-\int_{0}^{T} \int_{\mathrm{D}}^{T} \sum_{j=1}^{N_{s t}} \mathbf{N}_{j}(\mathbf{x}) i_{j}(t) \cdot \mathbf{A}^{\prime}(\mathbf{x}, t) \mathrm{dDd} t \\
=\int_{0}^{T} \int_{\mathrm{D}} \mathbf{H}_{\mathrm{fp}}(\operatorname{curl} \mathbf{A}(\mathbf{x}, t)) \cdot \operatorname{curl} \mathbf{A}^{\prime}(\mathbf{x}, t) \mathrm{dDd} t \\
\int_{0}^{T}\left[\frac{\mathrm{d}}{\mathrm{d} t} \int_{\mathrm{D}} \mathbf{A}(\mathbf{x}, t) \cdot \mathbf{N}_{j}(\mathbf{x}) \mathrm{dD}+R_{j} i_{j}(t)\right] \cdot \mathrm{i}^{\prime}(\mathrm{t}) \mathrm{dt}=\int_{0}^{\mathrm{T}} v_{j}(t) \cdot i_{j}^{\prime}(t) \mathrm{d} t j=1, . ., N_{s t}
\end{gathered}
$$

with $\mathbf{A}^{\prime}(\mathbf{x}, t)$ and $i_{j}^{\prime}(t)$ test functions which belong to the same functional spaces as $\mathbf{A}(\mathbf{x}, t)$ and $i_{j}(t)$ respectively.

\section{MODEL ORDER REDUCTION}

\section{A. Proper Generalized Decomposition}

To solve (6) and (7), the PGD method can be applied. The vector potential $\mathbf{A}(\mathbf{x}, t)$ is then approximated by a separated representation of space and time functions,

$$
\mathbf{A}(\mathbf{x}, t) \approx \sum_{j=1}^{M} \mathbf{R}_{j}(\mathbf{x}) S_{j}(t)
$$

with $M$ the number of modes of the expansion. The terms $\mathbf{R}_{n}(\mathbf{x}), S_{n}(t)$ and the currents $i_{l}(t)_{1 \leq l \leq N s t}$ in the $N_{s t}$ stranded inductors, are calculated iteratively. At the $n^{\text {th }}$ iteration, the approximation of the solution is $\mathbf{A}_{n}(\mathbf{x}, \mathrm{t})=\mathbf{R}_{n}(\mathbf{x}) S_{n}(t)+\mathbf{A}_{n-l}(\mathbf{x}, t)$ with $\mathbf{R}_{n}(\mathbf{x})$ and $S_{n}(t)$ the functions to determine belonging to $\mathbf{L}^{2}$ curl $(\mathrm{D})$ and $\mathrm{L}^{2}([0, T])$ and $\mathbf{A}_{n-1}(\mathbf{x}, t)$ the approximation determined during the previous $\mathrm{n}-1$ iterations. In (6), $\mathbf{A}(\mathbf{x}, t)$ is replaced by its approximation $\mathbf{A}_{\mathrm{n}}(\mathbf{x}, t)$. The test function is given by $\mathbf{A}^{\prime}(\mathbf{x}, t)=\mathbf{R}_{n}^{\prime}(\mathbf{x}) S_{n}(t)+\mathbf{R}_{\mathrm{n}}(\mathbf{x}) S_{n}{ }_{n}(t)$ with $\mathbf{R}_{n}^{\prime}(\mathbf{x})$ and $S_{n}{ }_{n}(t)$ test functions belonging to the same spaces as $\mathbf{R}_{n}(\mathbf{x})$ and $S_{n}(t)$. To calculate $\mathbf{R}_{n}(\mathbf{x}), S_{n}(t)$ and $i_{n}(t)_{1 \leq n \leq N s t}$, two sets of equations deduced from weak forms (6) and (7), are solved iteratively. First, we suppose that $S_{n}(t)$ and $i_{n}(t)_{1 \leq n \leq N s t}$ are known. Then, the test function becomes $\mathbf{A}^{\prime}(\mathbf{x}, t)=\mathbf{R}_{n}^{\prime}(\mathbf{x}) S_{n}(t)$ and $\mathbf{R}_{n}(\mathbf{x})$ is the solution of the weak formulation,

$$
\begin{aligned}
& A_{R} \int_{\mathrm{D}} v_{\mathrm{fp}} \operatorname{curl} \mathbf{R}(\mathbf{x}) \cdot \operatorname{curl} \mathbf{R}^{\prime}(\mathbf{x}) \mathrm{dD}= \\
& \sum_{j=1}^{N_{s t}} B_{R j} \int_{\mathrm{D}} \mathbf{N}_{j}(\mathbf{x}) \cdot \operatorname{curl} \mathbf{R}_{n}^{\prime}(\mathbf{x}) \mathrm{dD}+\mathbf{F}_{\mathrm{R}-\mathrm{L}}+\mathbf{F}_{\mathrm{R}-\mathrm{NL}} \\
& \text { with } A_{R}=\int_{0}^{T} S_{n}(t) \cdot S_{n}(t) \mathrm{d} t, B_{R j}=\int_{0}^{T} i_{j}(t) \cdot S_{n}(t) \mathrm{d} t, \\
& \mathbf{F}_{\mathrm{R}-\mathrm{L}}=-\int_{0}^{T} S_{n}(t) \int_{\mathrm{D}} v_{\mathrm{fp}} \operatorname{curl} \mathbf{A}_{n-l}(\mathbf{x}, t) \cdot \operatorname{curl} \mathbf{R}_{n}^{\prime}(\mathbf{x}) \mathrm{dDd} t, \\
& \mathbf{F}_{\mathrm{R}-\mathrm{NL}}=-\int_{0}^{T} S_{n}(t) \int_{\mathrm{D}} \mathbf{H}_{\mathrm{fp}}\left(\operatorname{curl} \mathbf{A}_{n}(\mathbf{x}, t)\right) \cdot \operatorname{curl}_{n}^{\prime}(\mathbf{x}) \mathrm{dDd} t
\end{aligned}
$$

In (9), the term $\mathbf{R}_{n}(\mathbf{x})$ is a function of $S_{n}(t)$ and $i_{l}(t)_{1 \leq l \leq N s t}$. We denote $\lambda$ the operator such that $\mathbf{R}_{n}(\mathbf{x})=\lambda\left(S_{n}(t), i_{l}(t)_{1 \leq l \leq N s t}\right)$. Secondly, to calculate the function $S_{n}(t)$ and update the currents $i_{l}(t)_{1 \leq l \leq N s t}$, we assume that the function $\mathbf{R}_{n}(\mathbf{x})$ is known. In this case, the test function in (6) is equal to $\mathbf{R}^{\prime}(\mathbf{x}) S_{n}(t)$. Considering (6) and (7), it can be shown that the functions $S_{n}(t)$ and $i_{l}(t)_{1 \leq l \leq N s t}$ are solutions of the following Ordinary Differential Equation (ODE) systems:

$$
\begin{aligned}
& A_{S} S_{n}(t)-\sum_{k=1}^{N_{S t}} B_{S k} i_{k}(t)=F_{S-L}+F_{S-N L} \\
& R_{k} i_{k}(t)+B_{S k} \frac{\mathrm{d} S_{n}(t)}{\mathrm{d} t}=v_{k}(t)+F_{i-k} \quad k \in\left\{1, \ldots, N_{s t}\right\} \\
& \text { with } A_{S}=\int_{\mathrm{D}} v_{\mathrm{fp}} \operatorname{curl} \mathbf{R}_{n}(\mathbf{x}) \cdot \operatorname{curl} \mathbf{R}_{n}(\mathbf{x}) \mathrm{dD}, \\
& B_{S k}=\int_{\mathrm{D}} \mathbf{N}_{k}(\mathbf{x}) \cdot \mathbf{R}_{n}(\mathbf{x}) \mathrm{dD}, \\
& F_{i-k}=-\sum_{j=1}^{n-l} \frac{\mathrm{d} S_{j}(t)}{\mathrm{d} t} \int_{\mathrm{D}} \mathbf{N}_{k}(\mathbf{x}) \cdot \mathbf{R}_{\mathrm{j}}(\mathbf{x}) \mathrm{dD} \\
& F_{S-L}=-\sum_{j=1}^{n-l} S_{j}(t) \int_{\mathrm{D}} v_{\mathrm{fp}} \operatorname{curl} \mathbf{R}_{j}(\mathbf{x}) \cdot \operatorname{curl}_{n}(\mathbf{x}) \mathrm{dD}, \\
& F_{S-N L}=\int_{\mathrm{D}} \mathbf{H}_{\mathrm{fp}}\left(\operatorname{curl} \mathbf{A}_{n}(\mathbf{x}, t)\right) \cdot \operatorname{curl} \mathbf{R}_{n}(\mathbf{x}) \mathrm{dD}
\end{aligned}
$$

Again, we define $\gamma$ an operator such that $\left(S_{n}(t)\right.$, $\left.i_{l}(t)_{1 \leq l \leq N s t}\right)=\gamma\left(\mathbf{R}_{n}(\mathbf{x})\right)$. The functions $\mathbf{R}_{n}(\mathbf{x}), S_{n}(t)$ and the currents $i_{l}(t)_{1 \leq l \leq N s t}$ are determined iteratively. At the $j^{\text {th }}$ iteration, assuming that $\left(S_{n}^{j}(t), i_{l}^{j}(t)_{1 \leq l \leq N s t}\right)$ are known, $\mathbf{R}_{n}^{j+1}(\mathbf{x})$ is given by $\mathbf{R}_{n}^{j+1}(\mathbf{x})=\lambda\left(S_{n}^{j}(t), i_{l}^{j}(t)_{1 \leq l \leq N s t}\right)$. Then, $\left(S_{n}^{j+1}(t), i_{l}^{j+1}(t)_{1 \leq l \leq N s t}\right)$ can be calculated by $\left(S_{n}^{j+1}(t), i_{l}^{j+1}(t)_{1 \leq l \leq N s t}\right)=\gamma\left(\mathbf{R}_{n}^{j+1}(\mathbf{x})\right)$. Finally, the solutions $\left(\mathbf{R}_{n}^{j}(\mathbf{x}), S_{n}^{j}(t), i_{l}^{j}(t)_{1 \leq l \leq N s t}\right)$ and $\left(\mathbf{R}_{n}^{j+1}(\mathbf{x}), S_{n}^{j+1}(\mathrm{t})\right.$, $\left.i_{l}^{j+1}(\mathrm{t})_{1 \leq l \leq N s t}\right)$ are compared. Once the solutions at $j^{\text {th }}$ and $(j+1)^{\text {th }}$ iterations are considered sufficiently close, one can proceed to the calculation of the next mode $n+1$. The operators $\lambda$ and $\gamma$ require the solution of (9) and (10) respectively. To solve (9), the field $\mathbf{R}_{n}(\mathbf{x})$ is approximated in the edge element space [12]. Then, we have ${ }_{\mathbf{R}_{n}}(\mathbf{x})=\sum_{l=1}^{N_{e}} R_{n, l} \mathbf{w}_{l}(\mathbf{x})$ with $R_{n, l}$ the circulation of

$\mathbf{R}_{n}(\mathbf{x})$ and $\mathbf{w}_{l}(\mathbf{x})$ the interpolation function associated with the $l^{\text {th }}$ edge and $N_{e}$ the number of degrees of freedom. The Galerkin method is applied to solve (9). The ODE (10) is solved using an implicit Euler scheme on $N_{T}$ time steps. 


\section{B. Discrete Empirical Interpolation Method}

We define a $N_{e} \times N_{T}$ matrix $\mathbf{M}_{\mathrm{fp}}$ of $N_{e} \times 1$ vectors $\mathbf{m}\left(t_{i}\right)_{1 \leq i \leq N T}$ such that their entries $m_{e}\left(t_{i}\right)$ satisfy:

$$
m_{e}\left(t_{i}\right)=\int_{\mathrm{D}} \mathbf{H}_{\mathrm{fp}}\left(\operatorname{curl} \mathbf{A}_{n}\left(\mathbf{x}, t_{i}\right)\right) \cdot \operatorname{curl} \mathbf{w}_{e}(\mathbf{x}) \mathrm{dD} \text { for } 1 \leq e \leq N_{e}
$$

It can be shown that the non-linear terms $\mathbf{F}_{\mathrm{R}-\mathrm{NL}}$ and $\mathrm{F}_{\mathrm{S}-\mathrm{NL}}$ can be expressed in function of the entries of the matrix $\mathbf{M}_{\mathrm{fp}}=\left(\mathbf{m}\left(t_{i}\right)\right)_{1 \leq i \leq N T}$. The entries of $\mathbf{M}_{\mathrm{fp}}$ must be evaluated for each new couple $\left(\mathbf{R}_{n}^{j}(\mathbf{x}), S_{n}{ }^{j}(t)\right)$. With a fine mesh and a large number of time steps $\mathrm{N}_{\mathrm{T}}$, the computation time of $\mathbf{M}_{\mathrm{fp}}$ can be prohibitive. To tackle this issue, an alternative is to use the Discrete Empirical Interpolation Method [5][6][13]. After each computation of $\mathbf{R}_{n}^{j}(\mathbf{x})$ and $S_{n}^{j}(t)$, the DEIM algorithm selects a small number $N_{D E I M}$ of most significant entries $m_{e}\left(t_{i}\right)$ (see (11)) of the vectors $\mathbf{m}\left(t_{l}\right)$ (called DEIM entries). Then, only $N_{D E I M}$ non-linear terms of $\mathbf{M}_{\mathrm{fp}}$ are computed and the other terms are interpolated to obtain an approximation of the matrix $\mathbf{M}_{\mathrm{fp}}$. To determine the DEIM entries, for a given mode $\mathrm{n}$ and an iteration $\mathrm{j}$, the field $\mathbf{A}_{n}(\mathbf{x}, t)$ is calculated for $N_{D E I M}$ time steps (for example, the $N_{\text {DEIM }}$ first time steps) from (8). We obtain then a $N_{e} \times N_{D E I M}$ matrix $\mathbf{M}_{\mathrm{s}}$ of the $\mathbf{m}\left(t_{l}\right)_{(1 \leq I \leq N D E I M)}$. The matrix $\mathbf{M}_{\mathrm{s}}$ is decomposed using a Singular Value Decomposition such as $\mathbf{M}_{\mathrm{s}}=\mathbf{V} \boldsymbol{\Sigma} \mathbf{W}$ with $\mathbf{V}_{N e \times N e}$ and $\mathbf{W}_{\text {NDEIM } \times N D E I M}$ orthogonal matrices and $\boldsymbol{\Sigma}_{\text {NeXNDEIM }}$ the diagonal matrix of the singular values. With the DEIM, only the $N_{m}$ most significant vectors $\mathbf{V}_{i}$ of the matrix $\mathbf{V}$ corresponding to the higher singular values of $\boldsymbol{\Sigma}$ are stored to construct a projector $\Psi\left(N_{e} \times N_{m}\right)$. Applying a greedy algorithm, a matrix $\mathbf{P}_{N e \times N m}$ composed of $N_{m}$ vectors of the identity matrix $\mathbf{I}_{N e \times N e}$ is determined from the indices of the most significant component of $\Psi$. We denote $I$ the set of these indices $I=\left(i_{1}, . ., i_{N m}\right)$. Then, for any time step $t_{l}$ in $[0, T]$, the vector $\mathbf{m}\left(t_{l}\right)$, and consequently the matrix $\mathbf{M}_{\mathrm{fp}}=\left(\mathbf{m}\left(t_{i}\right)\right)_{1 \leq i \leq N T}$, can be approximated by:

$$
\mathbf{m}\left(t_{l}\right) \approx \boldsymbol{\Psi}\left(\mathbf{P}^{\mathrm{t}} \boldsymbol{\Psi}\right)^{-1} \mathbf{m}_{\text {DEIM }}\left(t_{l}\right)
$$

with $\mathbf{m}_{\text {DEIM }}\left(t_{l}\right)$ the $N_{m} \times 1$ vector of entries $\left(m_{e}\left(t_{l}\right)\right)_{e \in I}$

\section{PGD_DEIM Model}

The strategy of the coupling between the PGD approach and the DEIM is given in the algorithm in Fig. 2. The internal loop ( $\mathrm{j}$ index) corresponds to the two steps for the computation of the functions $\mathbf{R}_{n}(\mathbf{x}), S_{n}(t)$ and $i_{l}(t)_{1 \leq l \leq N s t}$ (section III-A) and the approximation of the matrix $\mathbf{M}_{\mathrm{fp}}$ obtained from the DEIM (section III-B). The internal loop is stopped if the number of iterations is bigger than $I_{\max } n l$ or when the errors $\varepsilon_{n l-R}, \varepsilon_{n l-S}$ and $\varepsilon_{n l-i}$ on $\mathbf{R}_{n}(\mathbf{x}), S_{n}(t)$ and $i_{l}(t)_{1 \leq l \leq N s t}$ between two successive iterations are smaller than a criterion $\varepsilon_{n l}$. After each computation of a mode $\left(\mathbf{R}_{n}(\mathbf{x}), S_{n}(t)\right)$ and also of the updating currents $i_{l}(t)_{1 \leq l \leq N s t}$, an additional step can be added in order to recalculate all functions $S_{k}(t)_{1 \leq k \leq n}$ and $i_{l}(t)_{1 \leq l \leq N s t}$ to reduce the number of modes [2]. The external loop corresponds to the enrichment step ( $n$ index), this is stopped if the number of modes is reached or when the difference of the currents between two successive iterations is smaller than a criterion $\varepsilon$.

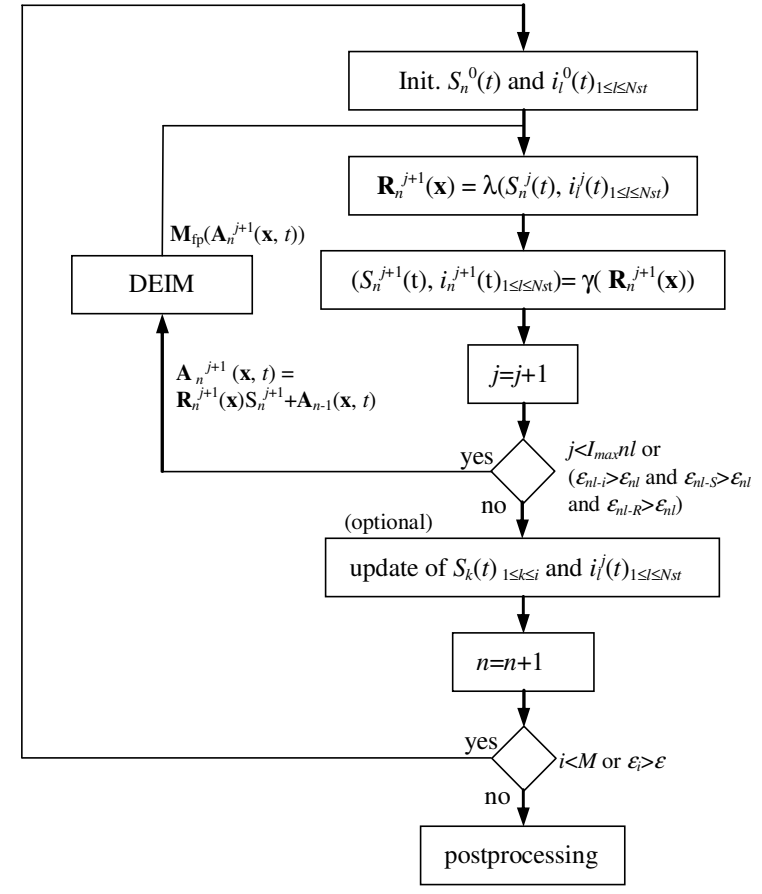

Fig. 2. Algorithm of the PGD_DEIM model

\section{ApPliCATION}

A 3D three phase EI transformer at no load is studied. Only one quarter of the transformer is modeled (Fig. 3.a) with the non-linear magnetic behavior of the iron core (Fig. 3.b). The 3D mesh is made of 12659 nodes and 67177 tetrahedra.
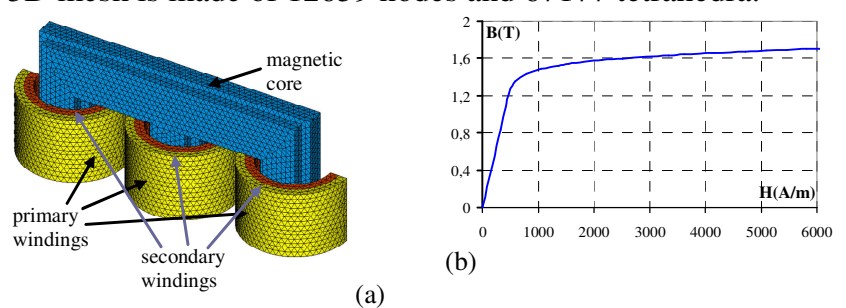

(b)

Fig. 3. Three phase EI transformer (a) and the $\mathrm{B}(\mathrm{H})$ curve of the ferromagnetic material (b)

First, the three phases of the transformer are supplied by sinusoidal voltages at a frequency equal to $50 \mathrm{~Hz}$. The time interval of simulation is fixed to [0;67 ms] with a time step of $67 \mu \mathrm{s}$. We compare the results obtained from the full model with those from a PGD_DEIM model where the first 40 time steps and the last 40 time steps $\left(N_{D E I M}=80\right)$ are used to approximate $\mathbf{M}_{\mathrm{fp}}(\mathbf{A}(\mathbf{x}, t))$ with the DEIM (see III-B). Figure 4 presents the error of the currents versus the number of modes. With 20 modes, the error is smaller than $2 \%$ for each current. Figure 5 compares the evolution of the currents obtained from reference and PGD_DEIM model at the beginning of the transient state where we can see a good agreement between the two models. In term of computation time, the full model and the PGD_DEIM model with 20 modes require $118 \mathrm{~min}$ and 56 min respectively. Then, the speed up is 2.1 . 


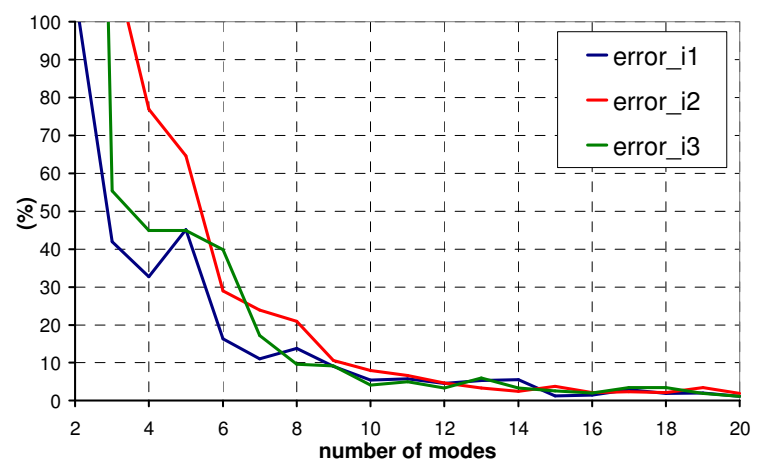

Fig. 4. Error of the currents as a function of the number of modes

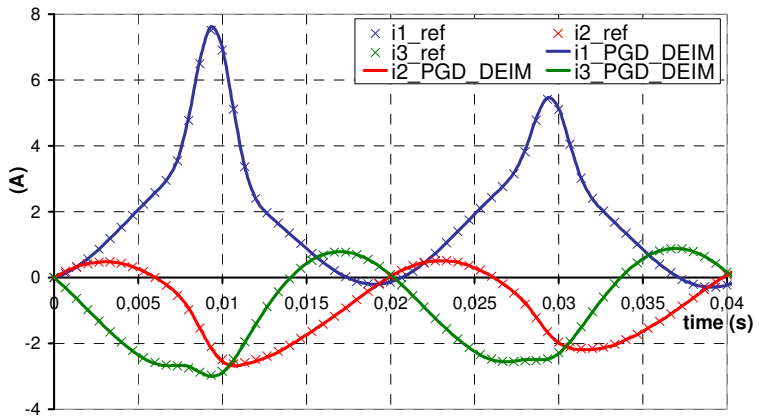

Fig. 5. Evolution of the currents obtained from reference and PGD_DEIM model at the beginning of the simulation

Now, the three phases of the transformer are supplied by 2level PWM voltages, and the carrier frequency is equal to 50 $\mathrm{Hz}$. The switching frequency is equal to $5 \mathrm{kHz}$. The time interval of simulation is fixed to [0;0.2 s] with a time step of $10 \mu \mathrm{s}$. In these conditions, the number of time steps is 20000. To limit the variation of the currents, an inductance is placed in series with each winding. For the DEIM, we select 50 vectors $\mathbf{m}\left(t_{i}\right)$ at the beginning and the end of the simulation interval every $0.5 \mathrm{~ms}$. It enables to cover the range of variation of the non-linear entries of $\mathbf{M}_{\mathrm{fp}}$. The evolutions of the error for the currents versus of the number of modes are presented in Fig. 6. With 12 modes, the error is smaller $0.5 \%$ for each current. Figure 7 presents the evolution of the currents obtained from reference and PGD_DEIM model at the beginning of the simulation. In term of computation time, the reference model and the PGD_DEIM model require $1510 \mathrm{~min}$ and $22 \mathrm{~min}$ respectively, the speed up is 26 .

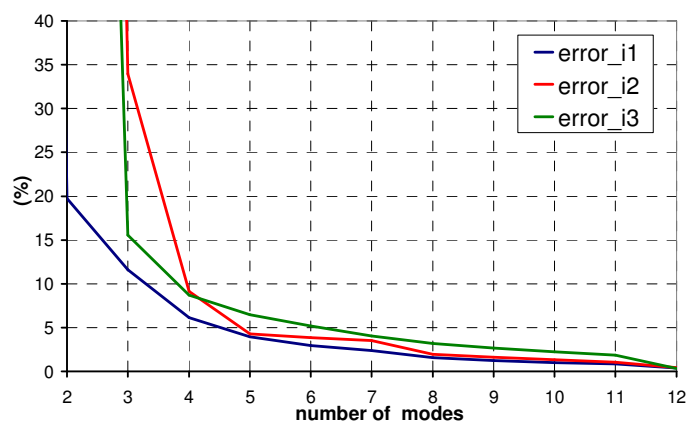

Fig. 6. Error of the currents as a function of the number of modes

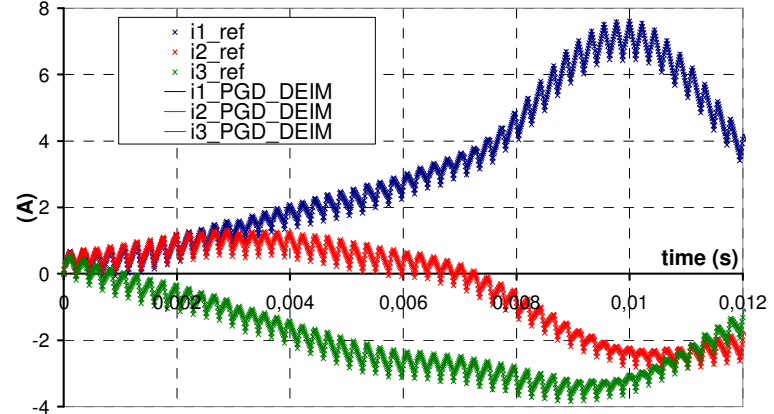

Fig. 7. Evolution of the currents obtain from reference and PGD_DEIM model at the beginning of the simulation

\section{CONCLUSION}

The Proper Generalized Decomposition method associated with the Discrete Empirical Interpolation Method has been applied to solve a 3D non-linear FE magnetostatic problem coupled with the circuit equations. The accuracy of the reduced model depends on the number of modes and the number of DEIM terms. On the studied example, it appears that the more the number of time step, the more the speed up between the PGD_DEIM model and the full model. This confirms the fact that the PGD seems to be very attractive when the number of time steps requires to be high.

\section{REFERENCES}

[1] J. Lumley, "The structure of inhomogeneous turbulence", Atmospheric Turbulence and Wave Propagation. A.M. Yaglom and V.I. Tatarski., pp. 221-227, 1967.

[2] T. Wittig, I. Munteanu, R. Schuhmann, and T. Weiland, "Two-step Lanczos algorithm for model order reduction," IEEE Trans. Magn., vol. 38, no. 2, pp. 673-676, Mar. 2002.

[3] F. Chinesta, A. Ammar, E. Cueto, "Recent Advances and New Challenges in the Use of the Proper Generalized Decomposition for Solving Multidimensional Models", Archives of Computational Methods in Engineering, vol. 17( 4), pp. 327-350, 2010.

[4] A. Nouy, "A priori model reduction through Proper Generalized Decomposition for solving time-dependent partial differential equations", Computer Methods in Applied Mechanics and Engineering, Elsevier, vol. 199(23-24), pp. 1603-1626, 2010.

[5] M. Barrault, N. C. Nguyen, Y. Maday, and A. T. Patera. "An "empirical interpolation"method: Application to efficient reduced-basis discretization of partial differential equations", C. R. Acad. Sci. Paris, vol. 339(9), 2004, pp. 667-672, 2004.

[6] S. Chaturantabut and D. C. Sorensen, "Nonlinear Model Reduction via Discrete Empirical Interpolation", SIAM J. Sci. Comput., vol. 32, no. 5, pp.2737-2764, 2010

[7] J. V. Aguado et al., "DEIM-Based PGD for parametric nonlinear model order reduction", VI International Conference on Adaptive Modeling and Simulation, ADMOS 2013, Lisbon (Portugal).

[8] P. Alotto, M. Guarnieri, F. Moro, A. Stella "A proper generalized decomposition approach for modeling fuel cell polymeric membranes" IEEE Trans. Mag., vol. 47( 5), pp. 1462-1465, 2011.

[9] T. Henneron, A. Benabou, S. Clénet, "Nonlinear Proper Generalized Decomposition Method Applied to the Magnetic Simulation of a SMC Microstructure", IEEE Trans. Mag., vol. 48(11), pp. 3242-3245, 2012.

[10] M. Pineda-Sanchez et al., "Simulation of skin effect via separated representations", COMPEL, vol. 29(4), pp.919-929, 2010.

[11] T. Henneron, S. Clénet, "Model order reduction of quasi-static problems based on POD and PGD approaches", Eur. Phys. J. Appl. Phys., vol. 64(2), 24514, 7 pages, 2013.

[12] A. Bossavit, "A rationale for edge-elements in 3-D fields computations", IEEE Trans. Magn., vol. 24(1), pp 74-79, 1988.

[13] T. Henneron, S. Clénet, "Model Order Reduction of Non-Linear Magnetostatic Problems Based on POD and DEI Methods", IEEE Trans. Mag., vol. 50(2), 2014. 